\title{
Regional probabilities of precipitation change: A Bayesian analysis of multimodel simulations
}

\author{
C. Tebaldi, ${ }^{1}$ L. O. Mearns, ${ }^{1}$ D. Nychka, ${ }^{2}$ and R. L. Smith ${ }^{3}$ \\ Received 16 August 2004; revised 16 August 2004; accepted 12 November 2004; published 28 December 2004.
}

[1] Tebaldi et al. [2005] present a Bayesian approach to determining probability distribution functions (PDFs) of temperature change at regional scales, from the output of a multi-model ensemble, run under the same scenario of future anthropogenic emissions. The main characteristic of the method is the formalization of the two criteria of bias and convergence that the REA method [Giorgi and Mearns, 2002] first quantified as a way of assessing model reliability. Thus, the General Circulation Models (AOGCMs) of the ensemble are combined in a way that accounts for their performance with respect to current climate and a measure of each model's agreement with the majority of the ensemble. We apply the Bayesian model to a set of transient experiments under two SRES scenarios. We focus on predictions of precipitation change, for land regions of subcontinental size. We highlight differences in the PDFs of precipitation change derived in regions where models find easy agreement, and perform well in simulating present day precipitation, compared to regions where models have large biases, and/or their future projections disagree. We compare results from the two scenarios, thus assessing the consequences of the two alternative hypotheses, and present summaries based on their averaging. INDEX TERMS: 1699 Global Change: General or miscellaneous; 6309 Policy Sciences: Decision making under uncertainty; 6334 Policy Sciences: Regional planning. Citation: Tebaldi, C., L. O. Mearns, D. Nychka, and R. L. Smith (2004), Regional probabilities of precipitation change: A Bayesian analysis of multimodel simulations, Geophys. Res. Lett., 31, L24213, doi:10.1029/ 2004GL021276.

\section{Introduction}

[2] The need of probabilistic assessment of climate change at regional scale has been clearly expressed in particular by the impacts research community [Reilly et al., 2001; Webster, 2003; Dessai and Hulme, 2003]. Output from AOGCMs is a basic ingredient of this research, since regional detail can be derived directly from the simulations and through dynamical or statistical downscaling [Wilks and Wilby, 1999; Giorgi, 1990]. It is recognized, however, that no model is the "true" model, and valuable information can be gathered from multi-model experiments, whose synthesis

\footnotetext{
${ }^{1}$ Institute for the Study of Society and the Environment, National Center for Atmospheric Research, Boulder, Colorado, USA.

${ }^{2}$ Geophysical Statistics Project, National Center for Atmospheric Research, Boulder, Colorado, USA.

${ }^{3}$ Department of Statistics, University of North Carolina, Chapel Hill, North Carolina, USA.
}

Copyright 2004 by the American Geophysical Union. 0094-8276/04/2004GL021276\$05.00 may provide a better estimate of the probability of climate change, particularly at regional scales.

[3] In Tebaldi et al. [2005] (hereinafter referred to as T05) we propose a statistical framework in which projections from different models contribute to a final PDF of climate change. The final estimates account for the relative performance of the models when simulating current climate (model bias), and the degree of inter-model consensus in their future (change) projections (model convergence). This way, we depart from previous studies as we model the ensemble members as realizations of random processes whose distributional characteristics inform the way of drawing inference. Previous work [Raisanen and Palmer, 2001] treated the ensemble members as equiprobable realizations, and determined probabilities of climate change by computing the fraction of ensemble members in which change exceeded specific thresholds. The differential quality of the members, in terms of each model's bias and convergence, was thus disregarded. Later Giorgi and Mearns [2003] incorporated the idea of a weighted average of the ensemble members in the counts of threshold exceedances. However, there is still a heuristic quality to this result, that our formulation avoids by positing statistical assumptions upfront, from which optimal estimation procedures follow.

[4] T05 is a methodological exposition, and uses temperature to exemplify the method's results. Here our method is used to combine signals of precipitation change from 9 AOGCMs run under two SRES scenarios [Cubasch et al., 2001], A2 and B2. We are interested in the results, rather than the method, and specifically in comparing PDFs across regions, seasons and scenarios.

\section{Methodology}

[5] T05 contains a detailed explanation of our method. Here we briefly summarize its most important aspects, in order to highlight the nature of Bayesian analysis in general, and the way our approach formalizes the bias and convergence criteria in combining multimodel projections.

[6] In a Bayesian framework all uncertain quantities are modeled as random parameters, with a prior probability distribution. In our case, two of these quantities are the present and future values of precipitation, aggregated into seasonal and regional 30-year means. Thus, for each region, $\mu$ and $\nu$ indicate respectively the true present and future precipitation, in winter, DJF, or summer, JJA. We assume prior distributions that are uninformative, i.e., Uniform distributions over $[0,+\infty)$. The data from observations and model output provide information to update the prior distribution into the posterior distribution, through Bayes' theorem. 
[7] The likelihood function specifies the distribution of the data as a function of the parameters. Thus, let $X_{0}$ be the observed value of precipitation in one region/season; $\lambda_{0}$ a measure of natural variability of observed precipitation; $X_{i}, i=1, \ldots, 9$ the current precipitation simulated by the 9 AOGCMs, $Y_{i}, i=1, \ldots, 9$ the corresponding future precipitation, and $\lambda_{1}, \ldots, \lambda_{9}$ measures of precision of each AOGCM in simulating regional precipitation, under the external forcings characterizing present and future climate runs. Note that the latter quantities are not observable but are modeled as random, so that a posterior distribution is derived for them as for the precipitation signals, $\mu$ and $\nu$ on the basis of the data. In T05 it is explained how the form of the joint posterior of $\mu, \nu, \lambda_{1}, \ldots, \lambda_{9}$ is approximated through Markov chain Monte Carlo (MCMC) simulation. MCMC algorithms generate sample values from the posterior, and allow accurate empirical estimation of its features. Here we also show analytical approximations of the posterior distribution shedding light on the nature of our statistical assumptions:

[8] 1 . The posterior mean of $\mu$ is approximately

$$
\tilde{\mu} \approx \frac{\sum_{0}^{9} \lambda_{i} X_{i}}{\sum_{0}^{9} \lambda_{i}},
$$

a weighted average of the observation and model output, with weights $\lambda_{0}, \lambda_{1}, \ldots, \lambda_{9}$.

[9] 2. The posterior mean of $v$ is approximately of the form

$$
\tilde{\nu} \approx \frac{\sum_{1}^{9} \lambda_{i} Y_{i}}{\sum_{1}^{9} \lambda_{i}},
$$

a weighted average of the 9 model responses, with weights $\lambda_{1}, \ldots, \lambda_{9}$.

[10] 3. $\Delta P=100 \cdot \frac{\nu-\mu}{\mu}$, the percent precipitation change, is a derived quantity and its posterior mean is similarly a weighted average of the individual models' precipitation change signals, with weights a function of $\lambda_{1}, \ldots, \lambda_{9}$.

[11] 4. Each $\lambda_{i}$ 's posterior mean is approximately

$$
\tilde{\lambda}_{i} \approx \frac{1}{\left|X_{i}-\tilde{X}\right|^{2}+\theta\left|Y_{i}-\tilde{Y}\right|^{2}}
$$

In (3) $\theta$ is an additional parameter of the likelihood function, acting as a multiplicative factor for the model precisions $\left(\lambda_{i}{ }^{\prime} \mathrm{s}\right)$ in the simulations of future climate. This allows for the possibility that models have a different degree of accuracy in simulating present and future climate. Thus, in (3) we formalize the idea of bias, through the expression $\left|X_{i}-\tilde{X}\right|$, measuring the distance between the $i$ th simulation of current precipitation and the "best approximation" (1) to the truth. The idea of convergence is quantified in the expression $\left|Y_{i}-\tilde{Y}\right|$, that measures the distance of the $i$ th future projection from the consensus estimate (2) of future precipitation. Since $\lambda_{i}$ can be interpreted as a measure of the $i$ th model's reliability, or accuracy, in simulating future and present climate, it follows from (3) and the forms of (1) and (2) that models with small bias and that agree with the
Table 1. Regions Used in the Analysis

\begin{tabular}{ll}
\hline Label & \multicolumn{1}{c}{ Region } \\
\hline NAU & North Australia \\
SAU & South Australia \\
AMZ & Amazons \\
SSA & Southern South America \\
CAM & Central America \\
WNA & West North America \\
CAN & Central North America \\
ENA & East North America \\
ALA & Alaska \\
GRL & Greenland \\
MED & Mediterranean \\
NEU & North Europe \\
WAF & West Africa \\
EAF & East Africa \\
SAF & South Africa \\
SAH & Sahara \\
SEA & South East Asia \\
EAS & East Asia \\
SAS & South Asia \\
CAS & Central Asia \\
TIB & Tibet \\
NAS & North Asia \\
\hline
\end{tabular}

consensus receive large weight in the posterior estimates of $\mu, \nu$ and $\Delta P$. In the standard version of our model the prior of $\theta$ is centered around 1 . This way the two criteria of bias and convergence are given a priori the same weight. If we wanted to discount the contribution of the convergence term in (3) relatively to the bias term, we could constrain the range of the prior for $\theta$ to values less than 1 , by which we would be modeling a larger variance for future than present projections.

[12] We apply our model to the precipitation data used by Giorgi and Mearns [2002]. Specifically, we analyse 22 land regions (see Table 1), two seasons (DJF and JJA) and two scenarios (A2 and B2). The nine AOGCMs are CCC, CCSR, CSIRO, GFDL, MPI, MRI, NCAR, NCAR-DOE and UKMO. The present-day average precipitation is defined as the mean of the 1961-1990 period (in simulations and observations), while the future averages are computed from the 2070-2099 period of the simulations. We make available code and data to reproduce our results on the Website http://www.cgd.ucar.edu/nychka/REA.

\section{Results}

[13] Figures 1 and 2 show boxplots of the posterior PDFs of percent precipitation change under the $\mathrm{A} 2$ and $\mathrm{B} 2$ scenario for DJF and JJA. The regions have been sorted by decreasing median change. Boxes extend from first to third quartile, medians are marked in each box, 'whiskers' cover the $95 \%$ probability interval. Points outside of this range are extreme values sampled by the MCMC simulation. For each region we show a pair of boxplots, the top referring to $\mathrm{A} 2$, the bottom to $\mathrm{B} 2$.

[14] There is large inter-regional variability, both in the central quartiles and in the ranges, with ALA in DJF and SAH in JJA showing very wide PDFs. The large spread is explained by large model biases (up to $340 \%$ for ALA, DJF and up to $600 \%$ for SAH, JJA) that translates into a high degree of uncertainty. In these two cases, the range of the PDFs extends beyond the range of the 9 AOGCM values. These results set our analysis apart from previous studies, 


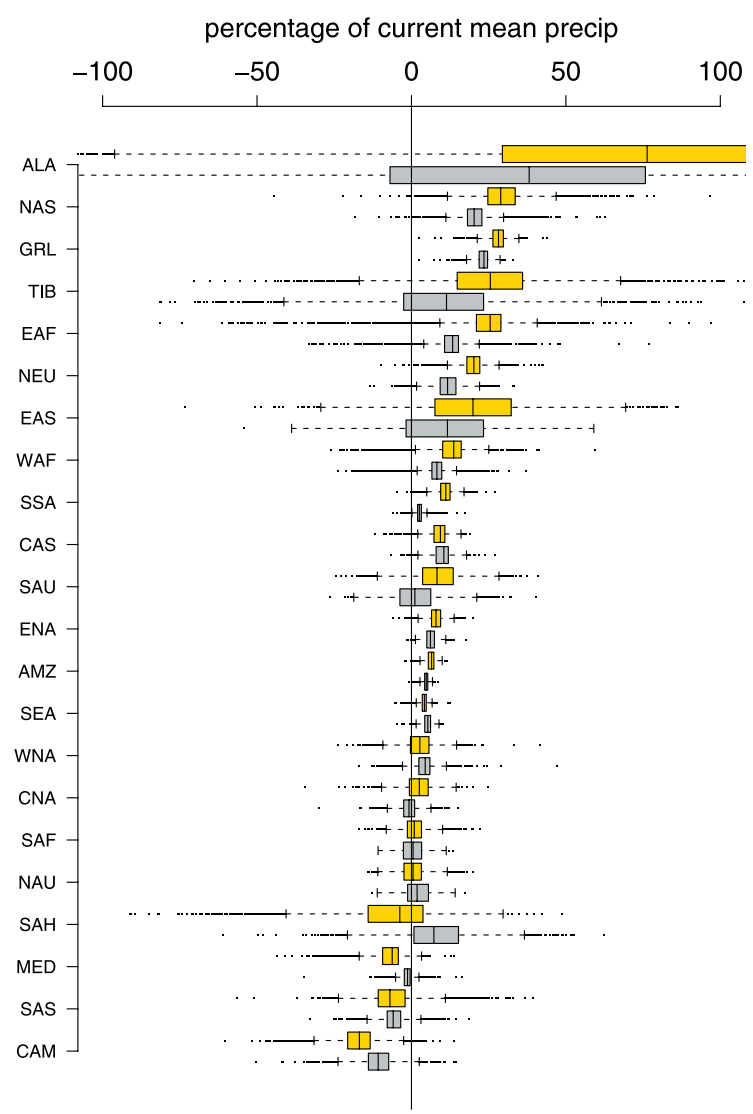

Figure 1. PDFs of percent precipitation change, DJF, for the 22 regions. For each region, top boxplot: change under A2; bottom: change under B2.

where the uncertainty is identified solely by the ensemble spread. The rest of the PDFs in Figures 1 and 2 are limited in range to a few tens of a percent in absolute value.

[15] When comparing the A2 and B2 scenarios, the majority of regions in both seasons show only a shift in the PDF ranges, and not a significant one (the two PDFs overlap in most cases), with the B2 scenario producing smaller changes of the same sign as A2. Only for SAH in DJF, under A2 the inter-quartile range is mostly negative, becoming mostly positive under B2. However, as a result of large biases, both PDFs show a large spread, i.e., a large degree of uncertainty.

[16] Figure 3 is similar to Figures 1 and 2, but now for each region a pair of boxplots show DJF and JJA percent precipitation changes, after averaging $\mathrm{A} 2$ and $\mathrm{B} 2$ by assigning them equal probability. We do so to provide a concise summary of the results, and justify this treatment in our specific case by the intermediate quality of A2 and B2, with respect to the full SRES spectrum, and by the linearity of the signal of precipitation change that we observed, when comparing the two scenarios. Given the necessary resources, a full probabilistic treatment of the SRES scenarios should ideally be undertaken.

[17] We order the regions according to decreasing median change in DJF. Again, the striking result is the large amount of variability in the extent of the boxes and their position relative to the zero line. Most of them lie on either side of the line, with just a few regions straddling it. For these regions the prediction is highly uncertain, assigning similar probability to negative and positive changes. For some of these regions the changes are relatively small: ALA, EAF, WAF, NEU in JJA and NAU, SAF,CNA in DJF have an interquartile range within $10 \%$ on either side of the zero line. For a few other the inter-quartile range extends beyond that in absolute value, indicating higher uncertainty. These are CAS in JJA and SAH in both seasons, although for JJA the mass of the PDF is shifted towards positive (and large) values, suggesting larger odds of precipitation increase.

[18] For the remaining regions positive changes are more prevalent than negative, especially in DJF. MED and CAM are an exception, concentrating over negative values for both seasons. NAU, SAF and CNA see a shift from "neutral" PDFs in DJF to negative values in JJA. The PDFs of all other regions shift from positive and large changes in DJF to either smaller but still positive changes in JJA (GRL, NAS, EAS, ENA, SSA) or neutral/negative changes (EAF, NEU, WAF, CAS, AMZ, SAU, WNA). The signal of change in SEA has almost identical PDFs in the two seasons (both very narrow, sign of a relatively sharper prediction) while SAS, contrary to the majority of regions, shows changes negative in DJF and positive in JJA.

[19] In summary, we find an extreme heterogeneity in the results, across regions and seasons, while we do not see significant differences when comparing the two SRES scenarios, with B2 just a weaker modulation of the A2 signal. A few region-season combinations result in very wide PDFs, a sign of highly uncertain predictions, a direct

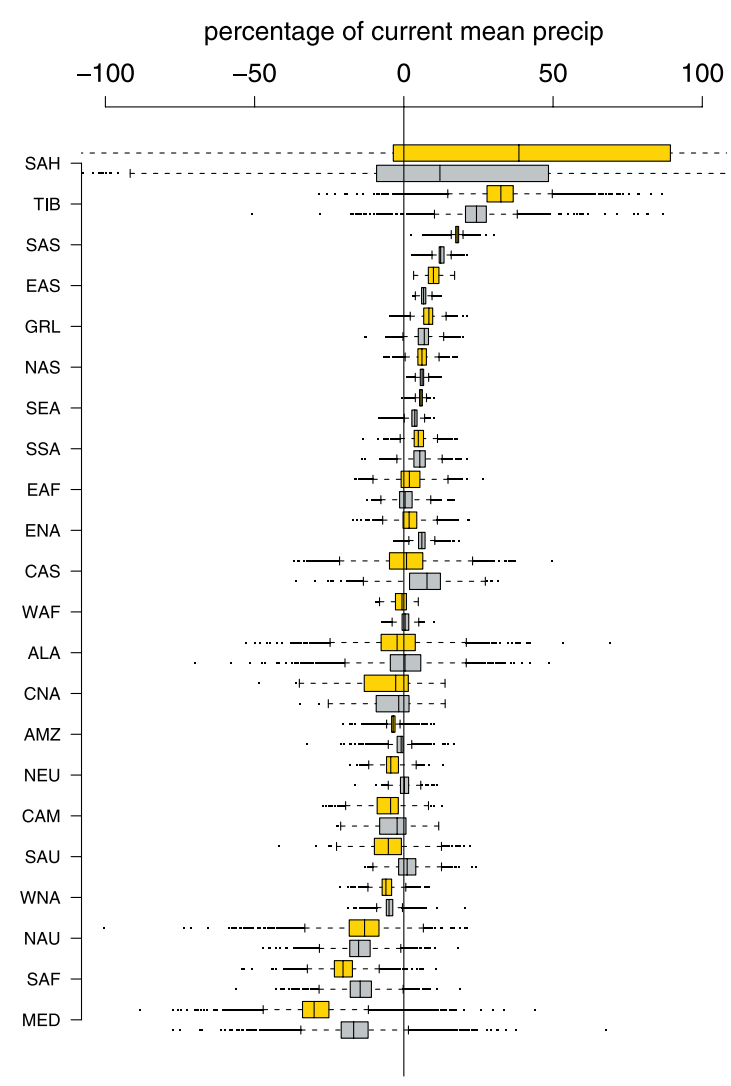

Figure 2. PDFs of percent precipitation change, JJA, for the 22 regions. For each region, top boxplot: change under A2; bottom: change under B2. 


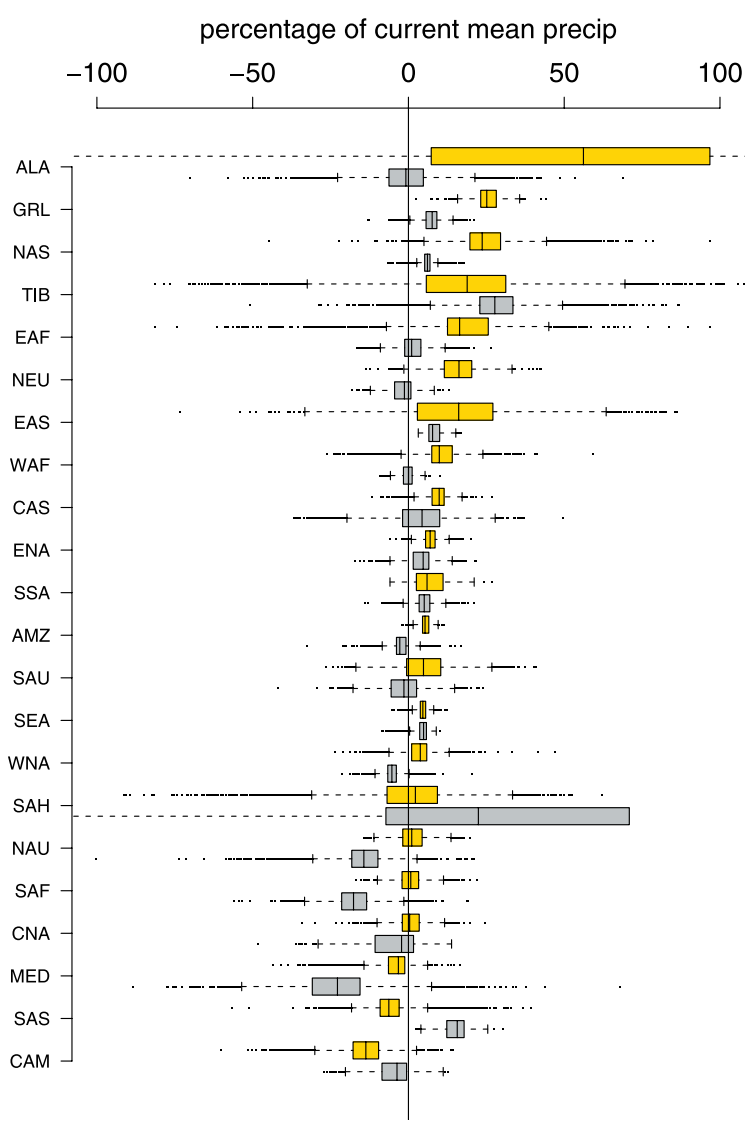

Figure 3. PDFs of percent precipitation change for the 22 regions. For each region, top boxplot: change in DJF; bottom: change in JJA. Distributions result from averaging over the A2 and B2 scenarios.

consequence of the large biases in the current climate simulations. For the majority of the regions a significant increase in the winter precipitation is predicted, often associated with smaller increases, not as significant, in summer. Only for a handful of regions the sign of the summer change is reversed. In a few cases the inter-quartile range straddles the zero line, thus making prediction of change uncertain not only in magnitude but in sign.

\section{Summary and Conclusions}

[20] We have applied the Bayesian model of T05 to a set of AOGCM experiments, and derived PDFs of precipitation change for 22 regions of sub-continental scale. Within the Bayesian framework we formalize two criteria of model evaluation, bias and convergence.

[21] The resulting PDFs of percent precipitation change show large inter-regional variability. For some regions PDFs are centered around zero, and two PDFs are particularly wide, encompassing both negative and positive changes, even larger than $100 \%$ in absolute value. Both are for areas where simulation of precipitation is particularly challenging, the Alaska and Sahara regions. For the majority of regions, though, the inter-quartile range is either above or below zero, suggesting significant change of a definite sign. An overall trend can be detected towards positive changes for
DJF and smaller in absolute value, sometime negative changes in JJA.

[22] Figure 4 shows the shapes of PDFs of precipitation change for 3 regions, the winter season and two alternative modeling choices, anticipated in Section 2. The curves drawn as solid lines result from the standard model. The dashed curves are derived by constraining the parameter $\theta$, through its prior, to assume values tightly concentrated around 0.1 . These 3 pairs of curves are indicative of general results. The effect of relaxing the convergence criterion, by effectively weighing it nine times less than the bias criterion, is to make the posterior PDFs wider, encompassing a larger fraction of the 9 AOGCMs results (shown as marks along the basis of the curves) rather than peaking over the regions where a subset of model projections converge. We note however that modes and high probability regions of the PDFs do not shift significantly.

[23] Figure 4 is also indicative of the general shape of the PDFs, unimodal and symmetric in most cases. An important role is played here by the natural variability parameter $\left(\lambda_{0}\right)$, that, when large, can modulate the impact of biases (see T05 for details). We find that for most region-season combinations, the natural variability is large enough to accommodate the 9 AOGCM biases, so that the performances of the 9 AOGCMs are comparable in terms of accuracy. As a result the weighting is fairly uniform among the individual
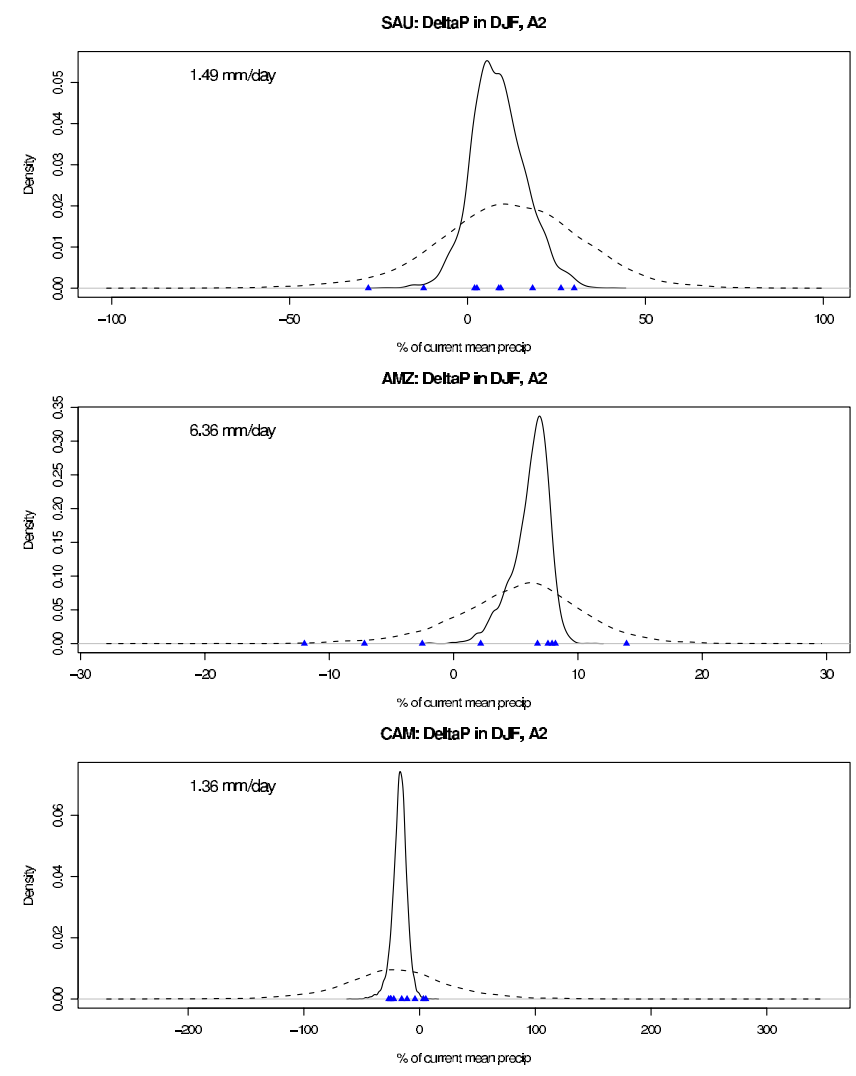

Figure 4. Examples of PDFs of percent precipitation change for 3 regions, in DJF, under SRES scenario A2. Solid lines: standard model, resulting in an equal a priori weight assigned to the two criteria; dashed lines: model where the convergence criterion is discounted, through a constrained prior on $\theta$. 
projections, and smooth curves are produced, encompassing the majority of projections.

[24] Acknowledgments. This research was supported through the National Center for Atmospheric Research Inititiative on Weather and Climate Impact Assessment Science, which is funded by the National Science Foundation. Additional support was provided through NSF grants DMS-0084375 and DMS-9815344. The authors thank an anonymous reviewer for constructive comments on an earlier version.

\section{References}

Cubasch, U., G. A. Meehl, G. J. Boer, R. J. Stouffer, M. Dix, A. Noda, C. A. Senior, S. Raper, and K. S. Yap (2001), Projections of future climate change, Climate Change 2001: The Scientific Basis: Contribution of Working Group I to the Third Assessment Report of the Intergovernmental Panel on Climate Change, edited by J. T. Houghton et al., pp. 525-582, Cambridge Univ. Press, New York.

Dessai, S., and M. Hulme (2003), Does climate policy need probabilities? Tyndall Cent. Working Pap., 34, Univ. of E. Anglia, Norwich, UK. (Available from http://www.tyndall.ac.uk/publications/publications. shtml.)

Giorgi, F. (1990), Simulation of regional climate using a Limited Area Model nested in a general circulation model, J. Clim., 3, 941-963.

Giorgi, F., and L. O. Mearns (2002), Calculation of average, uncertainty range and reliability of regional climate changes from AOGCM simula- tions via the "reliability ensemble averaging" (REA) method, J. Clim., $15,1141-1158$

Giorgi, F., and L. O. Mearns (2003), Probability of regional climate change based on the Reliability Ensemble Averaging (REA) method, Geophys. Res. Lett., 30(12), 1629, doi:10.1029/2003GL017130.

Raisanen, J., and T. N. Palmer (2001), A probability and decision model analysis of a multimodel ensemble of climate change simulations, J. Clim., 14, 3212-3226.

Reilly, J., P. H. Stone, C. E. Forest, M. D. Webster, H. D. Jacoby, and R. G. Prinn (2001), Uncertainty in climate change assessments, Science, 293, $430-433$

Tebaldi, C., R. W. Smith, D. Nychka, and L. O. Mearns (2005), Quantifying uncertainty in projections of regional climate change: A Bayesian approach to the analysis of multi-model ensembles, J. Clim., in press.

Webster, M. D. (2003), Communicating climate change uncertainty to policy-makers and the public, Clim. Change, 61, 1-8.

Wilks, D. S., and R. L. Wilby (1999), The weather generation game: A review of stochastic weather models, Prog. Phys. Geogr., 23, 329-357.

L. O. Mearns and C. Tebaldi, Institute for the Study of Society and the Environment, NCAR, P.O. Box 3000, Boulder, CO 80307, USA. (tebaldi@ucar.edu)

D. Nychka, Geophysical Statistics Project, NCAR, P.O. Box 3000, Boulder, CO 80307, USA

R. L. Smith, Department of Statistics, University of North Carolina, Chapel Hill, NC 27599, USA. 